\title{
4
}

\section{The Use of Near-Infrared Spectroscopy to Detect Differences in Brain Activation According to Different Experiences with Cosmetics}

\author{
Masayoshi Nagai ${ }^{1}$, Keiko Tagai ${ }^{2}$, Sadaki Takata ${ }^{2}$ and Takatsune Kumada ${ }^{1}$ \\ ${ }^{1}$ National Institute of Advanced Industrial Science and Technology (AIST) \\ ${ }^{2}$ Shiseido Co., Ltd. \\ Japan
}

\section{Introduction}

\subsection{Economic behavior and brain imaging}

Human economic and purchasing behavior has been studied primarily with brain imaging techniques such as functional magnetic resonance imaging (fMRI). One common topic in this domain of investigation is the willingness to pay (WTP). An essential component of every marketplace transaction is a WTP calculation in which buyers calculate the maximum amount of money they are willing to pay in exchange for the object being sold. WTP decisions are related to activation in the (medial) orbitofrontal cortex (OFC) (Wallis \& Miller, 2003; Padoa-Schioppa \& Assad, 2006; Erk, Spitze, Wunderlich, Galley, \& Walter, 2002; Rolls, 1996; Plassmann, O'Doherty, Rangel, 2007; Schoenbaum, Chiba, \& Gallagher, 1998; Tremblay \& Schultz, 1999; Roesch \& Olson, 2004). Medial OFC activation is related to choice or preference in the absence of explicit attention to the products presented, suggesting that the WTP calculation occurs implicitly. These studies have been performed under both wellcontrolled experimental settings and artificial environments because of the restrictions involved in using fMRI techniques. Although fMRI offers advantages related to the spatial resolution of cognitive and deep-brain activities, it requires a high degree of patient restraint during measurements made in pediatric research settings.

\subsection{Advantages of using near-infrared spectroscopy}

Near-infrared spectroscopy (NIRS) is a relatively new imaging technique that is noninvasive, highly flexible, and requires less participant restraint and sedation; it also produces continuous real-time measurements (Baird, Kagan, Gaudette, Walz, Hershlag, \& Boas, 2002; Endo, Nagai, Kumada, 2009; Kubota, Toichi, Shimizu, Mason, Findling, Yamamoto, \& Calabresea, 2006; Matsuda \& Hiraki, 2006; Otsuka, Nakato, Kanazawa, Yamaguchi, Watanabe, \& Kakigi, 2007). The NIRS technique has been successfully used in investigations of the neural correlates of complex actions such as walking and running on a treadmill (Suzuki, Miyai, Ono, Oda, Konishi, Kochiyama, \& Kubota, 2004), peeling an apple (Okamoto, Dan, Shimizu, Takeo, 
Amita, Oda, Konishi, Sakamoto, Isobe, Suzuki, Kohyama, \& Dan, 2004), demonstrating that NIRS can be applied to real-life situations involving actions and movements.

\subsection{In-store buying experiences and brain imaging}

Many different ways of trying articles/products at stores exist, including touching and listening to explanations offered by sales persons. However, the most effective ways to provide a good impression to customers or increase the chance that customers will purchase a given item remains unclear. Additionally, the association of the formation of an impression and buying behavior with brain activation has not been investigated. In actual situations, sales persons have a variety of ways to explain the features of articles to be sold. In the present study, we simulated a situation in which a customer (i.e., participant) and a sales person (i.e., confederate) interacted in five different ways.

\subsection{Purpose of the study}

The purpose of the current study was to investigate the relationship between different ways of learning about a cosmetic and brain activation and to examine the relationship between brain activation and impression formation and willingness-to-use decisions.

\section{Method}

\subsection{Participants}

Sixty women (age range: 25-39 years, average age: 30.6 years) participated in this experiment; the sample included 17 Japanese, 10 Chinese, 11 American, 11 German, and 11 Hispanic individuals. These classifications were based on nationality and place of residence. One left-handed subject (Hispanic) and two subjects (American and German) with a Japanese father or mother were excluded from the data analysis.

\subsection{Apparatus}

We used a multichannel NIRS system (ETG-4000, Hitachi Medical Corp., Tokyo, Japan) to measure brain activity. The NIRS unit was operated at near-infrared wavelengths of 695 and $830 \mathrm{~nm}$ and was used to measure temporal changes in concentrations of oxygenated hemoglobin (oxy-Hb), deoxygenated hemoglobin (deoxy-Hb), and total-hemoglobin. We used a pair of probe holders, each of which consisted of arrays measuring $4 \times 4$. Sixteen optodes (eight emitting and eight detecting) produced 24 channels for each probe. The distance between each emitting and corresponding detecting optode was $3.0 \mathrm{~cm}$. The sampling rate at each channel was approximately $10 \mathrm{~Hz}$.

The probes were placed on the frontal region of each hemisphere (Fig. 1). The $\mathrm{Cz}$ (international $10 / 20$ system) was set to align with a line connecting between the positions at the superior/posterior corners of the arrays (i.e., the emitter for the right probe and the detector for the left probe), and the $\mathrm{Fz}$ was aligned with the middle of the two connecting lines between the positions at the superior/anterior corners of the arrays and between the positions at the superior/second-from-anterior intersections of the arrays. Because the participant's heads were not exactly the same size, the Fz positions of participants differed slightly. The position of each column of emitters or detectors in the array was parallel to the midline of the brain. 


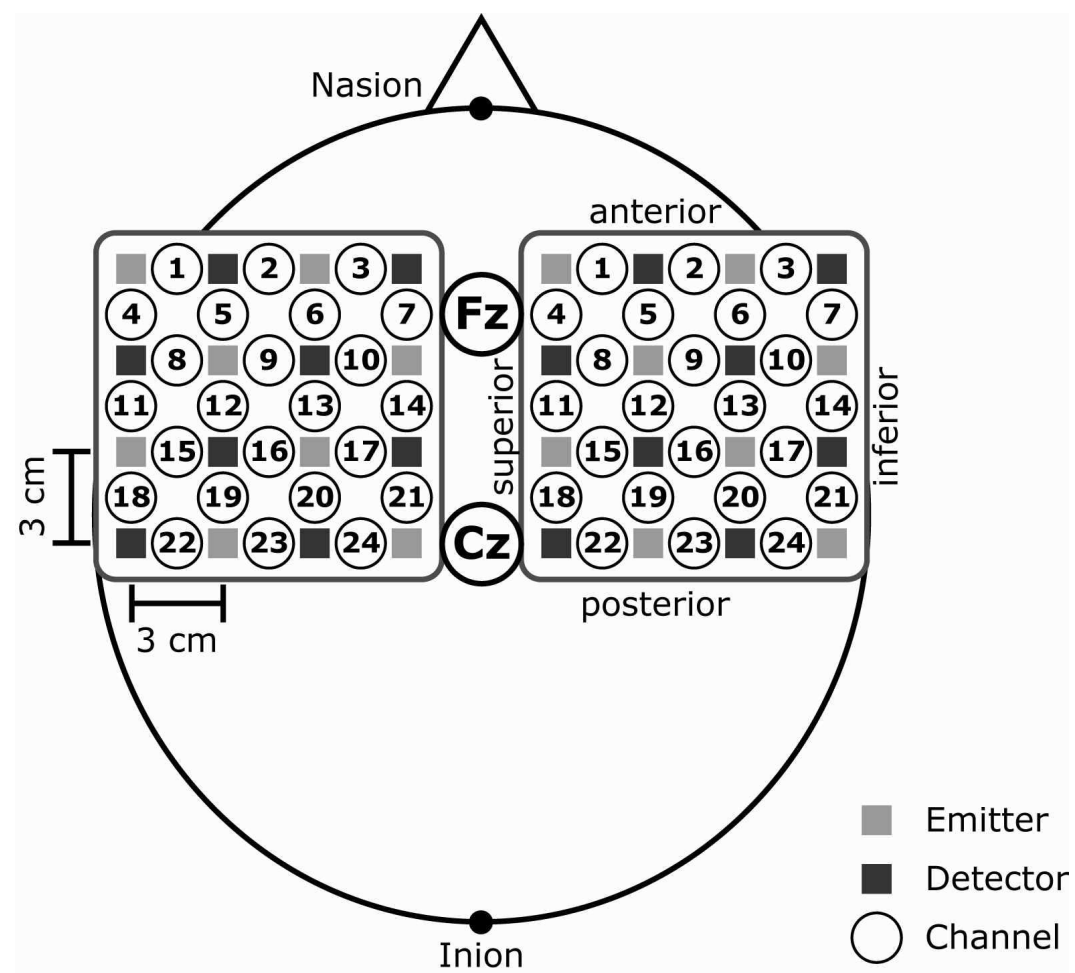

Fig. 1. Positions of optodes placed on the prefrontal regions of both hemispheres. The distance between each emitter (indicated by light gray squares) and the corresponding detector (indicated by dark gray squares) was $3 \mathrm{~cm}$. These emitters and detectors were placed in probes with $4 \times 4$ arrays.

\subsection{Stimuli, conditions, and task}

The task involved gathering information about a cosmetic article (facial cream) in five different ways (verbal explanation, written explanation, application, smell, and touch) in an environment simulating a cosmetics counter (Fig. 2). The facial cream was in a gold jar without a label bearing the name of a particular product. Five simulation conditions were used. Under the explanation condition, the subjects were asked to understand the explanation about the facial cream provided by a sales person (i.e., a confederate). Under the written condition, the explanation was given by a sales person, but he or she used a text and pictures to provide information about the facial cream. Under the application condition, the subjects were required to apply the facial cream to the back of their left hand. Under the smell condition, they were required to smell the facial cream on their left hand. Under the touch condition, they were required to touch a jar of facial cream. The order of these five conditions corresponded to the sequence above, and each condition lasted $15 \mathrm{sec}$. A 5-sec preparation time and a 35-sec rest time were added for NIRS data recording (Fig. 3). 


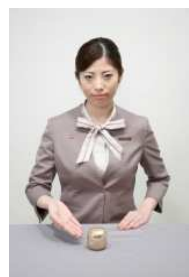

a)

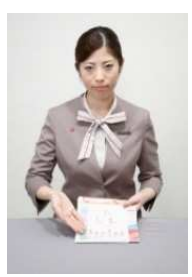

b)

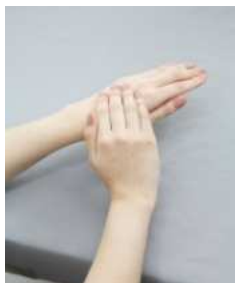

c)

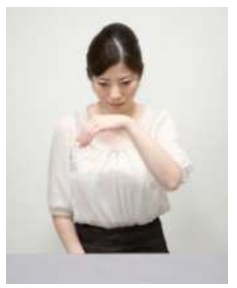

d)

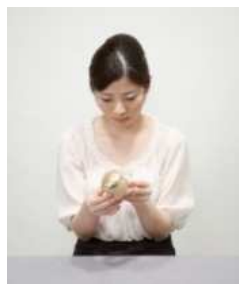

e)

Fig. 2. Five different ways by which participants learned about a facial cream: a) explanation, b) written, c) application, d) smell, and e) touch.

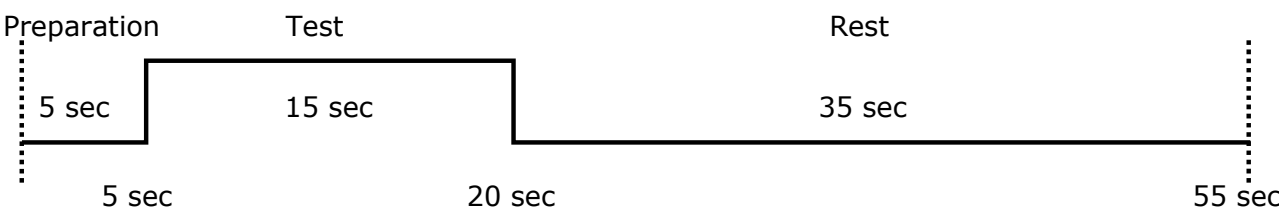

Fig. 3. Experimental schedule for NIRS recording

\subsection{Subjective rating}

After the NIRS measurements, subjects used a 100-point scale to rated the face cream with respect to 11 subjective reactions : 1 , comfort; 2 . relief; 3 , conviction; 4, satisfaction; 5, understanding; 6 , promise; 7 , reliability; 8 , likability; 9 , willingness to use; 10 . interest; and 11, appeal.

\section{Results}

\subsection{NIRS data analysis}

Trials that had movement artifacts, as reflected by steep changes in the time series of the $\mathrm{oxy}-\mathrm{Hb}$ and deoxy- $\mathrm{Hb}$ concentrations, were removed from the analysis. After these data were eliminated, we focused on oxy- $\mathrm{Hb}$ concentrations as the index of brain activation. Many NIRS studies use changes in oxy- $\mathrm{Hb}$ concentrations as the primary foci of analyses (e.g., Matsuda \& Hiraki, 2006; Otsuka, Nakato, Kanazawa, Yamaguchi, Watanabe, Kakigi, 2007) because these values are the most sensitive to changes in regional cerebral blood flow and are correlated with the blood-oxygen-level dependence (BOLD) signal (Hoshi, Kobayashi, \& Tamura, 2001; Strangman, Culver, Thompson, \& Boas, 2002).

Before starting the actual data analysis, the relative oxy-Hb concentrations at baseline (the line in the data repesenting oxy-Hb concentrations between the 5-sec preparation time and the last 5-sec rest time ) were calculated, which reduced low-frequency noise. Moreover, if the calculated relative oxy- $\mathrm{Hb}$ concentration values included an artifact, such as alternating current noise due to the poor contact of the probe with the skin, data from that channel under all conditions were deleted.

Data from one channel of one subject are shown in Fig. 4. The horizontal axis shows time from the beginning of the task, and the vertical axis shows oxy- $\mathrm{Hb}$ changes. This subject's 


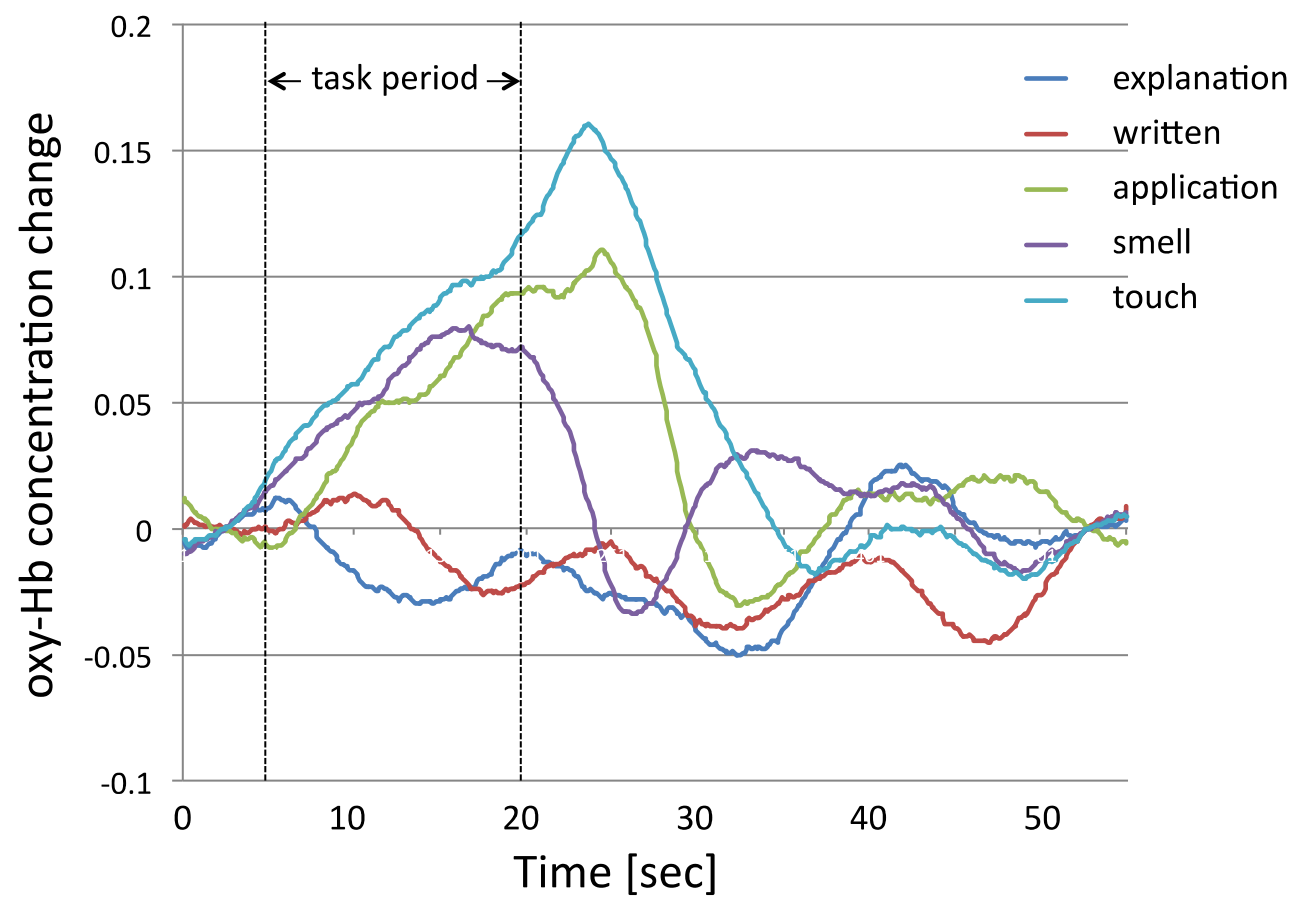

Fig. 4. An example of the time course of changes in the concentrations of oxy-hemoglobin $(\mathrm{Hb})$ under the five conditions.

data show that the oxy-Hb concentration increased after task onset and decreased after offset under the application, smell, and touch conditions. The brain-activation level was defined as the integral value of the oxy- $\mathrm{Hb}$ concentration during the task (15 sec). The average and standard deviation of the activation levels were calculated for each channel across subjects. When the activation level at a channel was higher than the average \pm 2.5 standard deviations, that channel's data were deleted from analysis under all conditions.

\subsection{Brain activation}

We performed $t$-tests to compare the activation levels with zero. Figure 5 shows the significantly activated channels under the five different conditions. The left and right 48 circles represent channel loci. Red, orange, and yellow circles show that the $p$-values for the test were $<0.01,0.05$, and 0.1, respectively. Following Okamoto, Dan, Sakamoto, Takeo, Shimizu, Kohno, Oda, Isobe, Suzuki, Kohyama, and Dan (2004), the channels were located in brain areas. One channel at the left frontal lobe was activated under the explanation condition. This channel is part of Broca's area, and its activation was due to the verbal explanation under the explanation condition. Under the written condition, the left and right superior frontal lobes, corresponding to the supplementary motor area, were activated. Under the written condition, participants needed to integrate the visual and auditory information on the form. These activated areas may have been related to integrated 

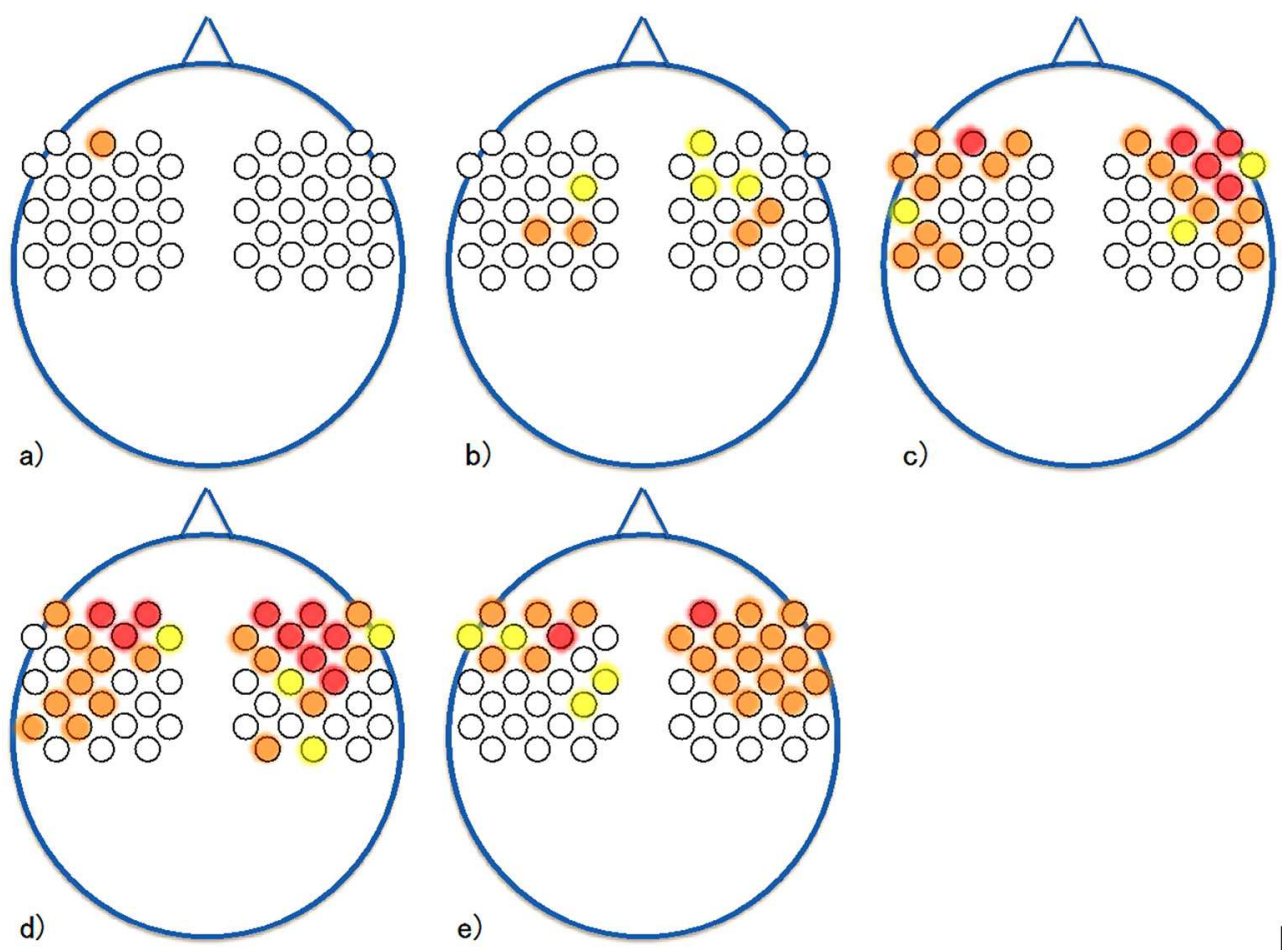

Fig. 5. Channels with statistically significant brain activation

processing. More channels and more areas were activated under the next three conditions than under the explanation and written conditions. Under the application condition, the left and right frontal and lateral lobes were activated. Under the smell condition, the strongest activation location shifted in the superior direction in the left hemisphere and activation in the right frontal area increased compared with that under the application condition. Moreover, activation in the lateral lobes was weakened compared with that under the application condition, and activation in the parietal lobes was also evident. Under the touch condition, activation in the left parietal lobe disappeared and activation in the right frontal lobe weakened compared with the results under the application condition. Under the latter three conditions, participants were required to perform motor actions and engage in sensory-information processing, which may have activated broader areas in the corresponding motor and sensory areas. These results indicate that different ways of considering a facial cream activated different brain areas.

\subsection{Brain activation and subjective ratings}

We analyzed the correlation between the subjective ratings and brain activation. The 11 subjective ratings were classified into two subtypes: "impressions" of the facial cream (1, comfort; 2 , relief; 3 , conviction; 4, satisfaction; 5 , understanding; 6 , promise; 7 , reliability; 8 , likability) and "willingness to use" (WTU) (9, willingness to use; 10, interest; 11, appeal.). 
Figure 6 shows the channels showing statistically significant correlations with impression or WTU. Under the explanation condition, brain activation in the left inferior frontal lobe was correlated with impression and WTU. Under the written condition, brain activation in the left parietal lobe was correlated with impression, and that in the right superior frontal lobe was correlated with impression and WTU. Under the application condition, brain activation in the left superior frontal lobe and lateral lobe was correlated with impression, that in the right superior frontal lobe was correlated with impression, and that in the right parietal and lateral lobes was correlated with impression and WTU. Under the smell condition, brain activation in the left parietal lobe was correlated with impression and WTU, that in the right inferior frontal lobe was correlated with impression, and that in the right parietal lobe was correlated with impression and WTU. Under the touch condition, brain activation in the left parietal lobe was correlated with impression, and that in the right parietal lobe was correlated with impression and WTU.
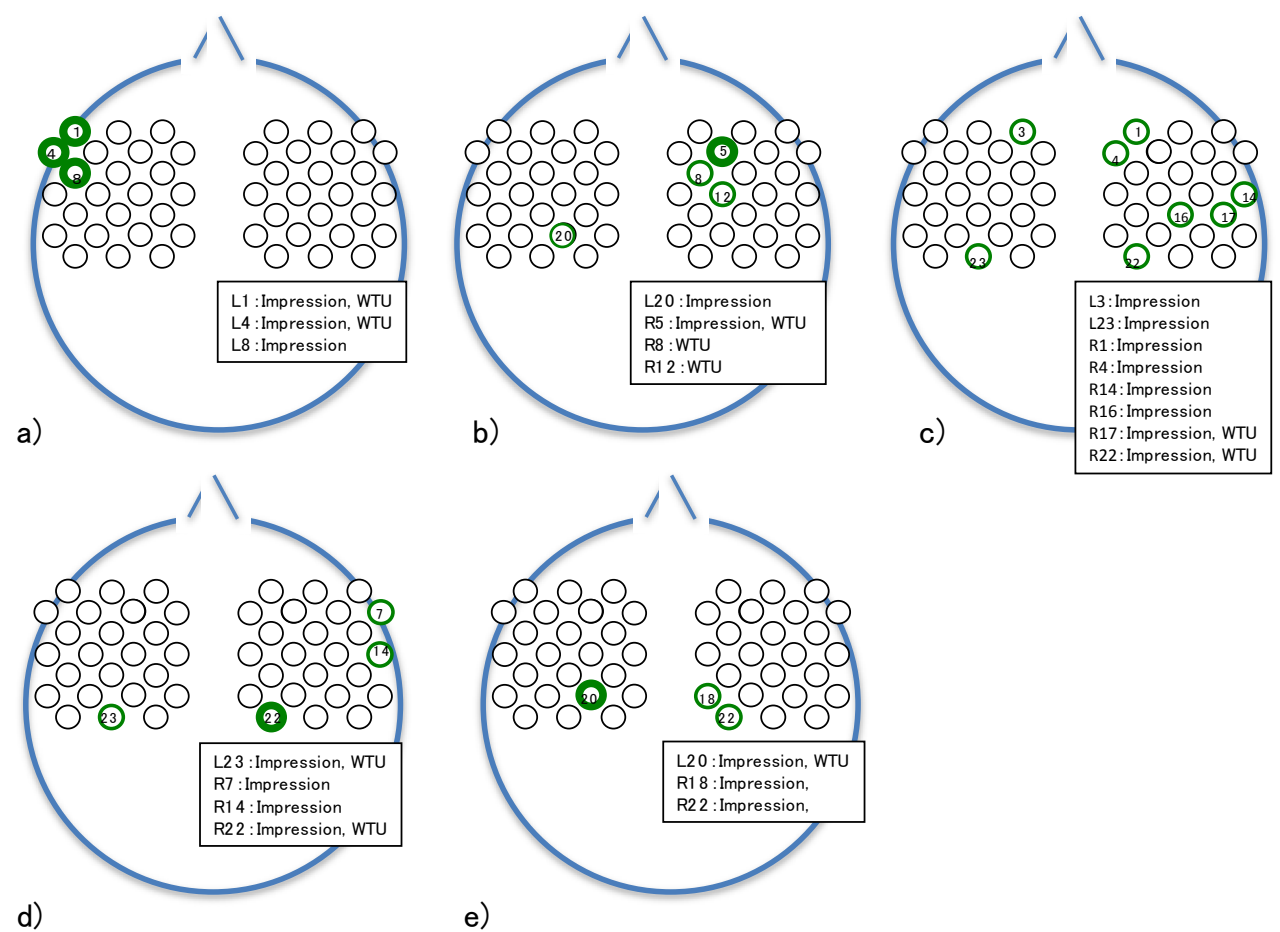

Fig. 6. Channels with statistically significant associations of brain activation with impression and WTU

\subsection{Brain activation and subjective ratings}

We analyzed the correlation between subjective ratings of the facial cream and brain activations using the dichotomy between impression and WTU described above.

We found the following relationships between brain activation and subjective ratings for impression and WTU. Under the explanation condition, brain activation in the left frontal 
lobe was correlated with impression and WTU; under the written condition, brain activation in the right superior frontal lobe was correlated with impression and WTU. Under the application condition, brain activation in the right superior frontal and parietal areas were related to impression and WTU. Under the smell and touch conditions, activation in the left and right parietal lobes was related to impression and WTU.

\subsection{Brain activation by subjective ratings and/or by task}

Three different trends emerged from comparisons among areas showing statistically significant activation while the subjects performed tasks (Fig. 5). Statistically significant correlations related to subjective rating are shown in Figure 6. The first trend involved the statistically significant relationship between channels and brain activation during tasks but did not include a correlation with subjective ratings. Most channels followed this trend (86\% of all channels). The second trend involved a statistically significant relationship between channel and brain activation during tasks and a significant correlation with subjective ratings. Only a few channels followed the second trend; one channel followed it under the written condition, two followed it under the smell condition, and five followed under the application condition. The third trend involved statistically non-significant relationships between channels and brain activation during a task but included significant correlations with subjective ratings. Thus, brain activations following the first and third trends showed stronger influences on subjective ratings.

\section{Discussion}

\subsection{Summary of results}

In the current experiment, participants learned about a facial cream in five different ways while their brain activity was monitored. They then rated their impressions and WTU the facial cream. The results suggested that different ways of learning about a facial cream activated different brain areas. Moreover, some channels showed a correlation between brain activation and subjective ratings.

\subsection{Relationship with WTP}

Many studies have suggested that WTP judgments are based on activation in the medial OFC. Because this area is not on the brain surface, it is difficult to monitor this activation with NIRS. We have shown that brain activation in the right superior frontal and the left and right parietal lobes was related to WTU. Because of the limitations of NIRS measurements, it is unclear whether the medial OFC was activated in the current experiments and related to WTU judgments.

\subsection{Difference by race}

Participants with different nationalities and different cultural backgrounds are expected to have different styles of thinking. A number of studies have suggested that people from different cultures have different cognitive processing styles (e.g., North Americans attend to focal objects more than do East Asians). Although these cultural differences may cause the correlations between brain activations and subjective ratings to differ among cultures, our 
results indicate the operation of a common brain mechanism in this regard. However, culture may nonetheless influence impressions, WTU, WTB, and brain activation related to these judgments. The cultural differences involved in-store shopping behaviors need to be understood given that such differences carry important implications for both academic and commercial domains.

\subsection{Advantage of NIRS for studying everyday situations}

The participants in the current study moved their arms and hands to apply a facial cream and touched jar. These actions are not permitted in magnetic resonance imaging scanners, and they also cause strong electroencephalogram artifacts due to muscle potentials. Thus, brain monitoring with NIRS is the only acceptable technique to use in situations in which participants move with relative freedom.

\section{Conclusion}

The current study was to investigate the relationship between different ways of learning about a cosmetic and brain activations. In the current experiment, participants learned about a facial cream in five different ways while their brain activity was monitored, and then rated their impressions and WTU on the facial cream. The results suggested that different ways of learning about a facial cream activated different brain areas. Moreover, some channels showed a correlation between brain activation and subjective ratings.

\section{References}

Baird, A. A., Kagan, J., Gaudette, T., Walz, K. A., Hershlag, N., \& Boas, D. A. (2002). Frontal lobe activation during object permanence: data from near-infrared spectroscopy. Neuroimage, 16:1120-5.

Endo, N., Nagai, M., \& Kumada, T. (2009). Objective estimation of state of content understanding by near-infrared spectroscopy (NIRS). Japanese Journal of Psychonomic Science, 28, 2-16.

Erk, S., Spitzer, M., Wunderlich, A., Galley, L., \& Walter, H. (2002) Cultural objects modulate reward circuitry. NeuroReport, 13:2499-2503

Hoshi, Y., Kobayashi, N., \& Tamura, M. (2001). Interpretation of near-infrared spectroscopy signals: a study with a newly developed perfused rat brain model. Journal of Applied Physiology, 90, 1657-1662.

Kubota, Y., Toichi, M., Shimizu, M., Mason, R. A., Findling, R. L., Yamamoto, K., \& Calabresea, J. R. (2006). Prefrontal hemodynamic activity predicts false memory - A near-infrared spectroscopy study. Neuroimage, 31, 1783-1789.

Matsuda, G., \& Hiraki, K. (2006). Sustained decrease in oxygenated hemoglobin during video games in the dorsal prefrontal cortex: a NIRS study of children. NeuroImage, 29, 706-711.

Okamoto, M., Dan, H., Shimizu, K., Takeo, K., Amita, T., Oda, I., Konishi, I., Sakamoto, K., Isobe, S., Suzuki, T., Kohyama, K., and Dan, I. (2004). Multimodal assessment of cortical activation during apple peeling by NIRS and fMRI. Neuroimage, 21, 12751288 . 
Otsuka, Y., Nakato, E., Kanazawa, S., Yamaguchi, M. K., Watanabe, S., \& Kakigi, R. (2007). Neural activation to upright and inverted faces in infants measured by near infrared spectroscopy. NeuroImage, 34, 399-406.

Padoa-Schioppa, C. \& Assad, J. A. (2006). Neurons in the orbitofrontal cortex encode economic value. Nature, 441:223-226.

Plassmann, H., O'Doherty, J. \& Rangel, A. (2007). Orbitofrontal cortex encodes willingness to pay in everyday economic transactions. Journal of Neuroscience. 27, 9984-9988.

Roesch, M. R. \& Olson, C. R. (2004). Neuronal activity related to reward value and motivation in primate frontal cortex. Science, 304, 307-310.

Schoenbaum, G., Chiba, A.A. \& Gallagher, M. (1998). Orbitofrontal cortex and basolateral amygdala encode expected outcomes during learning. Nature Neuroscience. 1, 155159.

Shibata, H., Suzuki, M., \& Gyoba, J. (2007). Cortical activity during the recognition of cooperative actions. Neuroreport, 7, 697-701.

Strangman, G., Culver, J. P., Thompson, J. H., \& Boas, D. A. (2002). A quantitative comparison of simultaneous BOLD fMRI and NIRS recordings during functional brain activation. NeuroImage, 17, 719-731.

Suzuki, M., Miyai, I., Ono, T., Oda, I., Konishi, I., Kochiyama, T., \& Kubota, K. (2004). Prefrontal and premotor cortices are involved in adapting walking and running speed on the treadmill: an optical imaging study. Neuroimage, 23, 1020-1026.

Tremblay L. \& Schultz W. (1999) Relative reward preference in primate orbitofrontal cortex. Nature, 398, 704-708.

Wallis, J. D. \& Miller, E. K. (2003) Neuronal activity in primate dorsolateral and orbital prefrontal cortex during performance of a reward preference task. European Journal of Neuroscience, 18, 2069-2081. 


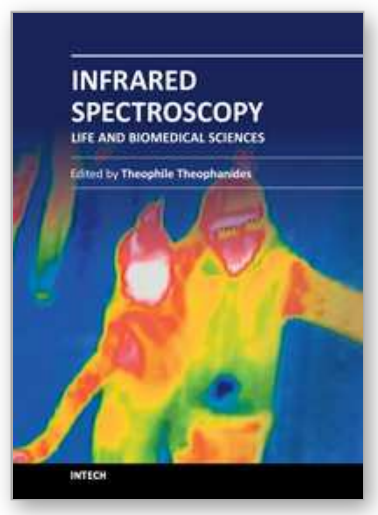

\author{
Infrared Spectroscopy - Life and Biomedical Sciences \\ Edited by Prof. Theophanides Theophile
}

ISBN 978-953-51-0538-1

Hard cover, 368 pages

Publisher InTech

Published online 25, April, 2012

Published in print edition April, 2012

This informative and state-of-the art book on Infrared Spectroscopy in Life sciences designed for researchers, academics as well as for those working in industry, agriculture and in pharmaceutical companies features 20 chapters of applications of MIRS and NIRS in brain activity and clinical research. It shows excellent FT-IR spectra of breast tissues, atheromatic plaques, human bones and projects assessment of haemodynamic activation in the cerebral cortex, brain oxygenation studies and many interesting insights from a medical perspective.

\title{
How to reference
}

In order to correctly reference this scholarly work, feel free to copy and paste the following:

Masayoshi Nagai, Keiko Tagai, Sadaki Takata and Takatsune Kumada (2012). The Use of Near-Infrared Spectroscopy to Detect Differences in Brain Activation According to Different Experiences with Cosmetics, Infrared Spectroscopy - Life and Biomedical Sciences, Prof. Theophanides Theophile (Ed.), ISBN: 978-953-510538-1, InTech, Available from: http://www.intechopen.com/books/infrared-spectroscopy-life-and-biomedicalsciences/the-use-of-near-infrared-spectroscopy-to-detect-differences-in-brain-activation-according-to-differe

\section{INTECH}

open science | open minds

\section{InTech Europe}

University Campus STeP Ri

Slavka Krautzeka 83/A

51000 Rijeka, Croatia

Phone: +385 (51) 770447

Fax: +385 (51) 686166

www.intechopen.com

\section{InTech China}

Unit 405, Office Block, Hotel Equatorial Shanghai

No.65, Yan An Road (West), Shanghai, 200040, China

中国上海市延安西路65号上海国际贵都大饭店办公楼405单元

Phone: +86-21-62489820

Fax: +86-21-62489821 
(C) 2012 The Author(s). Licensee IntechOpen. This is an open access article distributed under the terms of the Creative Commons Attribution 3.0 License, which permits unrestricted use, distribution, and reproduction in any medium, provided the original work is properly cited. 